\title{
Bone marrow infiltrated natural killer cells predicted the anti-leukemia activity of MCL1 or BCL2 inhibitors in acute myeloid leukemia
}

Yu-Jun Dai ${ }^{1,2^{*}+}$ DD, Si-Yuan He ${ }^{3+}$, Fang Hu ${ }^{1,2+}$, Xue-Ping $\mathrm{Li}^{1,2+}$, Jian-Ming Zhang ${ }^{4}$, Si-Liang Chen ${ }^{5}$, Wei-Na Zhang ${ }^{6}$, Hai-Min Sun ${ }^{7,8}$ and Da-Wei Wang ${ }^{4,7^{*}}$ (D)

\begin{abstract}
Acute myeloid leukemia (AML) is still incurable due to its heterogeneity and complexity of tumor microenvironment. It is imperative therefore to understand the molecular pathogenesis of AML and identify leukemia-associated biomarkers to formulate effective treatment strategies. Here, we systematically analyzed the clinical characters and natural killer (NK) cells portion in seventy newly-diagnosis (ND) AML patients. We found that the proportion of NK cells in the bone marrow of ND-AML patients could predict the prognosis of patients by analyzing the types and expression abundance of NK related ligands in tumor cells. Furthermore, MCL1 inhibitor but not BCL2 inhibitor combined with NK cell-based immunotherapy could effectively improve the therapeutic efficiency via inhibiting proliferation and inducing apoptosis of AML primary cells as well as cell lines in vitro. There results provide valuable insights that could help for exploring new therapeutic strategies for leukemia treatment.
\end{abstract}

Keywords: NK cells, MCL1 inhibitor, BCL2 inhibitor, AML, Immunotherapy

\section{Main text}

Natural killer (NK) cells are a type of cytotoxic immune cells that can recognize and kill cancer cells rapidly and efficiently. In recent years, more and more studies have revealed the biological characteristics of NK cells and their ability to recognize cancer cells directly [1]. Although immunotherapy has made a great breakthrough in stimulating the immune system against hematologic malignancies, there are few studies on NK cell-based immunotherapy [2].

\footnotetext{
* Correspondence: daiyj@sysucc.org.cn; wdw12114@rjh.com.cn

${ }^{+}$Yu-Jun Dai, Si-Yuan He, Fang Hu and Xue-Ping Li are joint first authors 'Department of Hematologic Oncology, Sun Yat-sen University Cancer Center, Guangzhou 500020, China

${ }^{4}$ National Research Center for Translational Medicine, Ruijin Hospital affiliated to Shanghai Jiao Tong University School of Medicine, Shanghai 200025, China

Full list of author information is available at the end of the article
}

Killer cell immunoglobulin-like receptors (KIR) are a type of receptor mainly expressed on the surface of human NK cells and partially activated T cells [3]. Functionally, KIR genes could be divided into inhibitory and activated types, which can specifically recognize and bind HLA class I molecules on the surface of target cells. They regulate the killing function of active cells in an effective switch system and play an important role in anti-infection and anti-tumor [4].

In this study, we elucidated the clinical relevance of KIRs and NK cells in bone marrow (BM) of acute myeloid leukemia (AML) patients. Our data indicated that NK cell ratio can predict the prognosis of patients, and can synergistically kill leukemia cells with MCL1 inhibitors to improve treatment efficiency.

(c) The Author(s). 2021 Open Access This article is licensed under a Creative Commons Attribution 4.0 International License, which permits use, sharing, adaptation, distribution and reproduction in any medium or format, as long as you give appropriate credit to the original author(s) and the source, provide a link to the Creative Commons licence, and indicate if changes were made. The images or other third party material in this article are included in the article's Creative Commons licence, unless indicated otherwise in a credit line to the material. If material is not included in the article's Creative Commons licence and your intended use is not permitted by statutory regulation or exceeds the permitted use, you will need to obtain permission directly from the copyright holder. To view a copy of this licence, visit http://creativecommons.org/licenses/by/4.0/. The Creative Commons Public Domain Dedication waiver (http://creativecommons.org/publicdomain/zero/1.0/) applies to the data made available in this article, unless otherwise stated in a credit line to the data. 


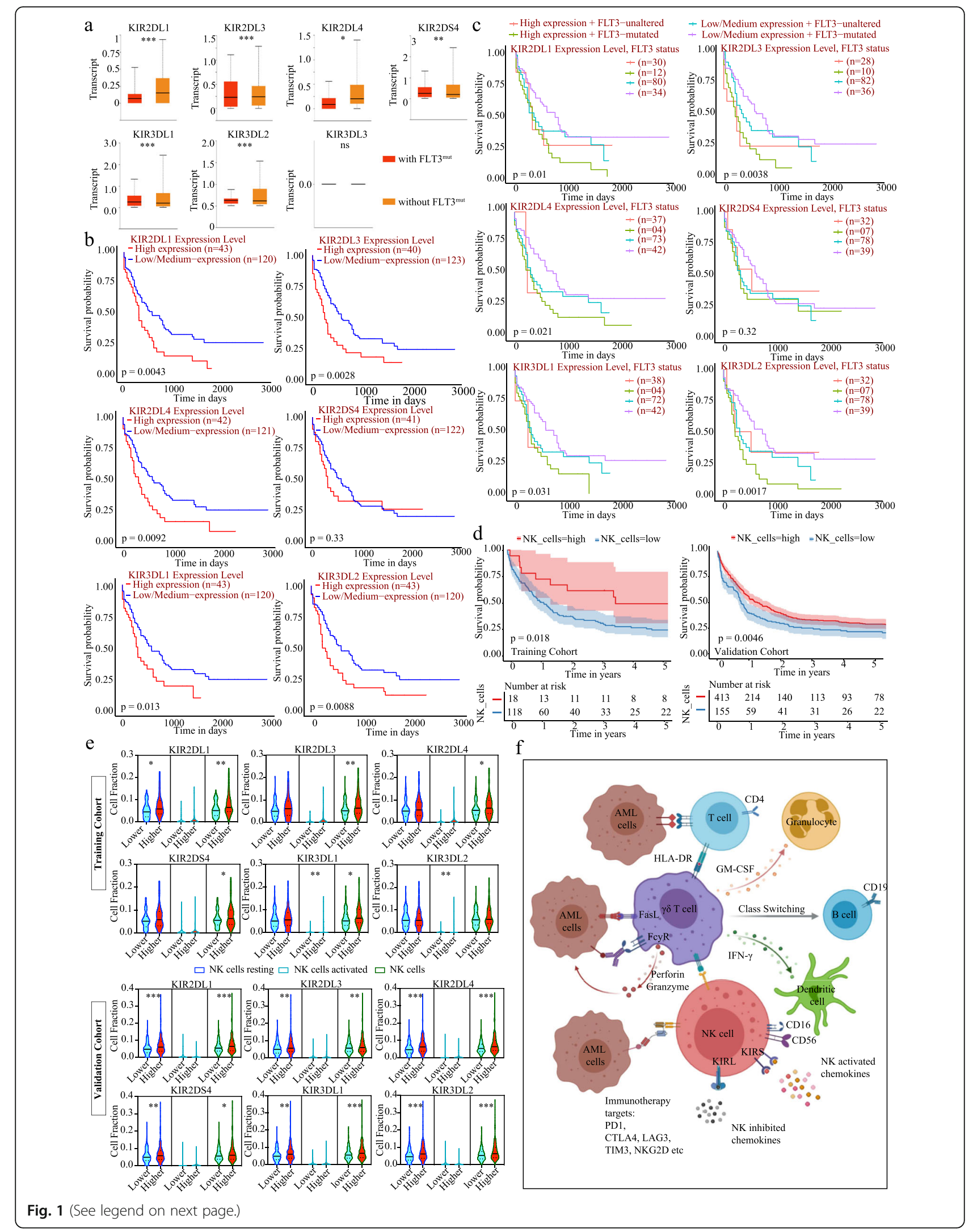


(See figure on previous page.)

Fig. 1 Overall survival (OS) of AML patients based on their BM infiltrated NK cells and its receptors KIRs. a KIRs expression levels in patients with or without FLT3 mutations. b Kaplan-Meier plots of OS for patients according to the expression level of KIRs, respectively. c The prognostic values of the KIRs factors combined with FLT3 mutation status. $\mathbf{d}$ OS of patients according to the BM infiltrated NK cells in training (4 missing cases) and validation cohorts (10 missing cases). e The expression of KIRs were in groups with high or low NK cells, activated and resting NK cells, respectively. Resting NK cells is colored in blue, activated NK cells in Turquoise, and bulk NK cells in green. Mean \pm SEM values are shown. ${ }^{*} P<$ 0.05 , ${ }^{*} P<0.01,{ }^{* *} P<0.001$. f A proposed cellular model to describe the function of immune cells in patients

\section{Results and discussions}

\section{Expression level and prognosis of KIRs in AML}

Here we reported a study on the transcriptional levels of KIRs in cancer and normal samples by analyzing the data from Oncomine and ENCORI (The Encyclopedia of RNA Interactomes) (Additional file 1 and Table S1). These results indicated that KIRs played an important role in solid tumors such as kidney renal clear cell carcinoma and lung cancer. In AML patients, the expression levels of KIRs were much higher than that in normal samples (Additional file 2). Notably, the expression of KIR2DL group (KIR2DL1, KIR2DL3 and KIR2DL4) was significantly downregulated in patients with FLT3 mutations, whereas KIR2DS and KIR3DL group (except KIR3DL3) were upregulated (Fig. 1a). Interestingly, RAS activation status was not related to the expression of KIRDS in AML (Additional file 3). Next, we further explored the critical efficiency of KIRs for predicting the survival of patients with AML. The Kaplan-Meier curve and log rank test analyses revealed that the increased KIR2DL1 $(p=0.0043)$, KIR2DL3 $(p=0.0028), \operatorname{KIR} 2 D L 4(p=0.0092)$, KIR3DL1 $(p=0.013)$ and KIR3DL2 $(p=0.0088)$ mRNA levels were significantly related to poor prognosis for overall survival (OS) of AML patients (Fig. 1b). Whereas, the KIR2DS4 mRNA level had no tendency to indicate prognosis $(P=0.33)$. Furthermore, when FLT3 mutation status was combined, the prognostic values of the KIRs factors were consistent with the above results (Fig. 1c).

\section{NK cells in BM indicated poor prognosis in AML}

The immune dysfunction recently has been considered as a risk factor in AML and predicted poor prognosis [5]. Next, we utilized Cibersort to deconvolute the geneexpression data of 713 newly diagnosed AML (NDAML) patients (140 patients as Training cohort and 573 patients as Validation cohort) and generated a gene matrix with a signature of more than 10 immune cell subtypes (Table S2 and S3). Patients were divided into "low" and "high" subgroups (according to the cutoff value of conversion score of total NK cells, activated NK cells and resting NK cells, respectively). The prognostic analysis indicated that the low NK cells or low resting NK cells predict poor prognosis, while the low activated NK cells indicated a favorable prognosis in AML patients (Fig. 1d and Additional file 4). Notably, the differential expression of KIRs were only in total NK cells but not in activated or resting NK cells (Fig. 1e). Thus, we proposed a model to describe the cellular and molecular basis for the potential prognostic value of monitoring the proportion of immune cells in patients (Fig. 1f).

Further, the single-cell RNA sequence data of AML patients at diagnosis and matched samples after chemotherapy were used for immune cell subtype analysis [6]. UMAP (Uniform Manifold Approximation and Projection) analysis indicated the proportion of NK cells in total BM cells of AML samples at diagnosis was much lower than those in matched samples after chemotherapy (Fig. 2a). We further validated the NK cells proportion $\left(\mathrm{CD} 45^{+} \mathrm{CD}^{-} \mathrm{CD}^{-} 6^{+} \mathrm{CD} 16^{+}\right)$of lymphocytes in $\mathrm{BM}$ cells from patients with hematological malignancies $(30$ lymphoma cases without BM infiltration as control; 95 ND-AML cases and 25 refractory/relapse (R/R) AML cases) (Fig. 2b). The proportion of lymphocytes, especially NK cells in BM, was significantly decreased in ND-AML samples compared with normal samples, and the ratio reached the lowest in R/R AML cases (Fig. 2c). These data were consistent with theory that NK cells might be one of important mediators of anti-leukemia immunity and indicated that NK cells of BM might play an anti-leukemia effect in leukemogenesis [7, 8]. In addition, KIRs expressions were much higher in $R / R$ AML samples among these three groups (Fig. 2d and Table S4). When we divided ND-AML samples into high NK cells group and low NK cells group, according to the proportion of NK cells in BM. We found there was no significant differences in the clinical characteristics of the two groups (Table 1). However, KIRs were mainly expressed in samples with low NK cell group (Fig. 2e and Table S4). The expression data further validated survival data of NK cells and KIRs expression patterns which showed above.

\section{NK cells could synergy with MCL1 inhibitor to kill leukemia cells}

Previous studies showed that BCL-2 and MCL-1 proteins could control the survival of NK cells in vivo [9]. Thus, we explored the relationship between the expression of these two molecules and NK cells in BM. We found MCL1 expression level has a significant negative 


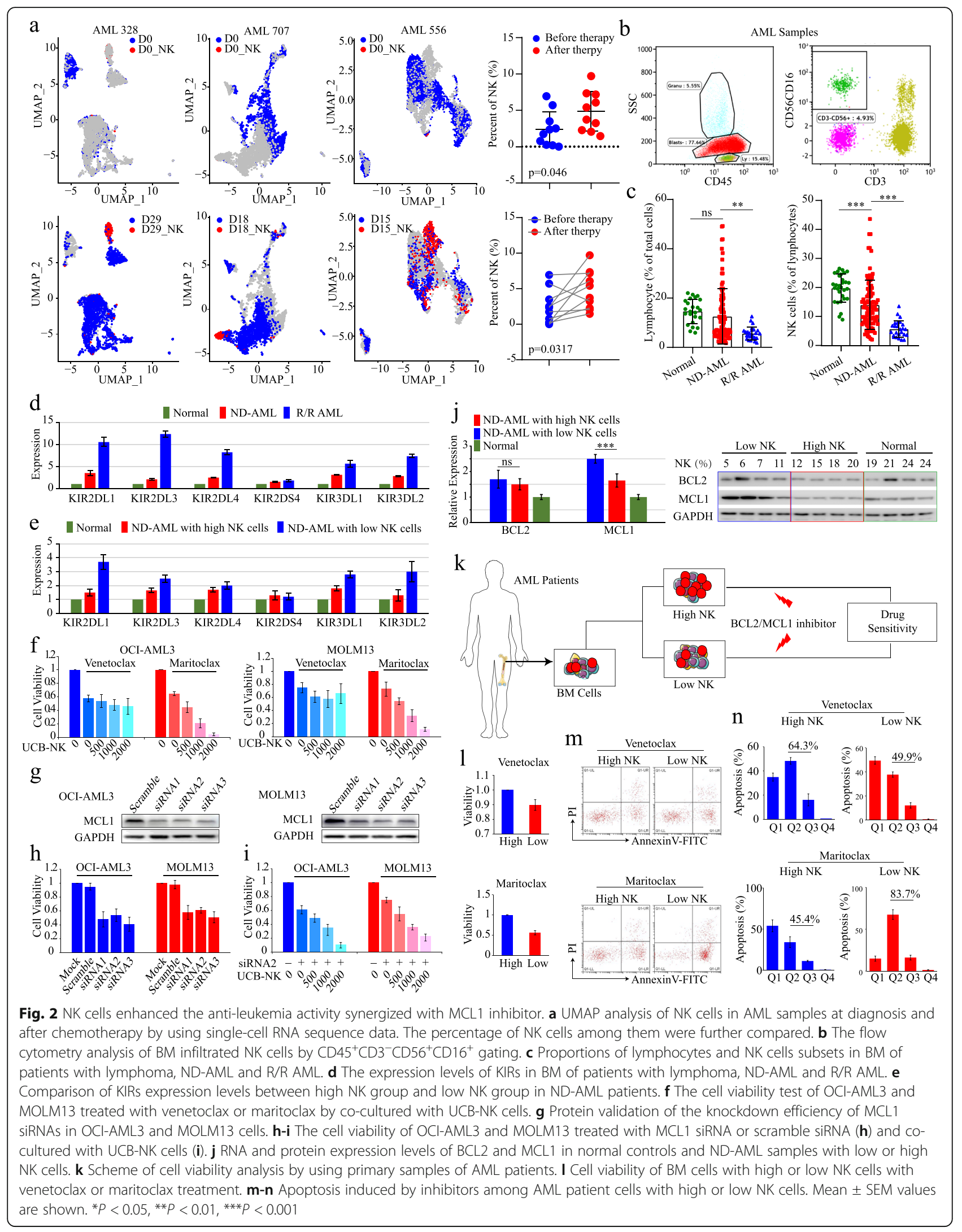


Table 1 Basic characteristics of ND-AML patients between NK\% high and NK\% low in BM

\begin{tabular}{llc}
\hline Variable & ND-AML NK\% & Nigh \\
\hline Age, years & & N4) \\
$\quad$ Median & & 47 \\
$\quad$ Range & $15-78$ & $19-74$ \\
Gender, no. (\%) & & \\
$\quad$ Male & $20(58.8 \%)$ & $16(45.7 \%)$ \\
Female & $14(41.2 \%)$ & $19(54.3 \%)$
\end{tabular}

Hepatic or renal function, no. ${ }^{2}$

$9(54.3 \%)$

Normal

Abnormal

WBC, $10^{9} / \mathrm{L}$

Median

Range

Platelet, $10^{9} / \mathrm{L}$

Median

Range

$\mathrm{HB}, \mathrm{g} / \mathrm{L}$

Median

Range

PB NK, \%

Median

Range

BM blasts, \%

Median

Range

BM Lymph, \%

Median

Range

BM NK, \%

Median

Range

BM T, \%

Median

Range

Cytogenetics, no. (\%)
t(15;17)/PML-RARA
t(8;21)/AML1-ETO
inv.(16;16)/CBFb-MYH11
CN-AML ${ }^{b}$
Unfavorable $^{c}$

Mutations, no. (\%)
32

5.4

2.3-21.7

53.1

16.3-95.1

6.2

1.7-49.2

$P$ value

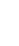
FLT3 mutations
15
5
RAS mutations
6
6

ALT Alanine aminotransferase, AST Aspartate aminotransferase, WBC White blood cell, RBC Red blood cell, $H B$ Hemoglobin, $B M$ Bone marrow, ns Not significance

$P$ values were calculated by means of nonparametric Wilcoxon rank-sum test for continuous variables and $\mathrm{x}$-square test for categorical variables ${ }^{a}$ Hepatic abnormality as defined by ALT 2.53 normal value or AST 2.53 normal value, while renal abnormality as defined by creatinine 2.53 normal value ${ }^{b} \mathrm{CN}-\mathrm{AML}$ : cases having no cytogenetically identifiable abnormalities

'Unfavorable: inv. (3)/t (3;3), t (9;22), 11 q23 abnormalities, 25, 27, del (5q),del (7p), and complex karyotype 
correlation with NK cells, while BCL2 expression level has no correlation with NK cells both in training and validation cohorts (Additional file 5). As we known, BCL2 and MCL1 inhibitors are currently considered as new therapeutics for leukemia, and showed promising response in patients [10]. Therefore, we quest whether NK cells could synergize with these new inhibitors to achieve better therapeutic effects. To test our hypothesis, we treated AML cell lines OCI-AML3 and MOLM13 with BCL2 inhibitor (Venetoclax) or MCL1 inhibitor (Maritoclax), then co-cultured with gradient numbers of NK cells derived from umbilical cord blood (UCB-NK). The cell viability suggested that UCB-NK could cooperate with maritoclax but not venetoclax to kill leukemia cells (Fig. 2f). Moreover, we applied the MCL1 siRNA to knockdown the expression of MCL1 in OCI-AML3 and MOLM13 cells and then cocultured with UCB-NK (Fig. 2g-i). The cell viability was significantly decreased in both OCI-AML3 and MOLM13 cells treated with MCL1 siRNA compared with those treated with scramble siRNA (Fig. $2 \mathrm{~h}$ ). In addition, the cell viability of OCIAML3 and MOLM13 was significantly impaired after treatment with MCL1 siRNA and UCB-NK cells (Fig. 2i). This result was consistent with the data on the pharmacological effects of maritoclax (Fig. 2f). Next, we sought to validate effects by using mononuclear cells isolated from the BM of ND-AML patients. Samples were divided into two groups according to the NK proportion in BM (Fig. 2k and Table S4), and the RNA/protein expression levels of BCL2 and MCL1 were examined. MCL1 was much more expressed in the low NK cell group of ND-AML samples both at transcriptional and translational level (Fig. 2j). Consistent with the results from cell line studies, patient samples with low NK treated with maritoclax were less viable than samples with high NK (Fig. 2l). In addition, maritoclax induced cell apoptosis was significant enhanced in samples with low NK proportion (Fig. $2 \mathrm{~m}$ and $\mathrm{n}$ ). While there was no significant difference in cell viability and apoptosis between samples with high NK and low NK with venetoclax treatment (Fig. 2k-n).

\section{Conclusions}

The importance of NK ratio in BM of AML patients has generated tremendous interest in understanding its role in disease management. Immune dysfunction could predict therapeutic reactivity and unfavorable prognosis [5]. By analyzing the types and expression abundance of ligands related to NK function expressed in tumor cells, we first proposed that the proportion of NK cells in the $\mathrm{BM}$ of AML patients could predict the prognosis of patients. The lower the proportion of NK cells, the worse of the prognosis. Therefore, increasing the number of NK cells through small molecule compounds or cytokines, such as interleukin 15 or interleukin 2 etc., may be a new method of anti-leukemia. Next, we plan to perform experiments to verify the effect of NK on prognosis in different conditions, and conduct prospective controlled cohort clinical trials to verify this hypothesis in the future. Second, our data indicated that NK cellbased immunotherapy combined with MCL1 inhibitor but not BCL2 inhibitor could effectively improve the therapeutic efficiency of AML. These findings might provide new insights and theoretical basis for exploring new targets for leukemia treatment.

\section{Supplementary Information}

The online version contains supplementary material available at https://doi. org/10.1186/s12943-020-01302-6.

\section{Additional file 1. \\ Additional file 2. \\ Additional file 3. \\ Additional file 4. \\ Additional file 5. \\ Additional file 6. \\ Additional file 7. \\ Additional file 8. \\ Additional file 9.}

Additional file 10.

\begin{abstract}
Abbreviations
NK: Natural killer; KIR: Killer cell immunoglobulin-like receptors; HLA: Human leukocyte antigen; ENCORI: The Encyclopedia of RNA Interactomes; AML: Acute Myeloid Leukemia; ND-AML: Newly diagnosed AML; UMAP: Uniform Manifold Approximation and Projection; R/R AML: Refractory/ relapse AML; UCB-NK: NK cells derived from umbilical cord blood
\end{abstract}

\section{Acknowledgments}

We especially thank Xiang-Qin Weng (Ruijin Hospital affiliated to Shanghai Jiao Tong University School of Medicine) for performing flow cytometric analysis of AML samples.

\section{Authors' contributions}

Contribution: Y.J.D. designed and performed all cell culture experiments, interpreted data, created a graphical summary, and wrote the paper; F.H. and S.L.C performed bioinformatics analyses; S.Y.H performed single-cell sequencing analysis interpreted data; J.M.Z wrote the paper; F.H., X.P.L., W.N.Z. and H.M.S. assisted cell culture experiments and edited the paper; D.W.W. conceived and oversaw the study, interpreted data, and wrote the paper. The author(s) read and approved the final manuscript.

\section{Funding}

This work was supported by National Natural Science Foundation of China (82000144 and 81800140), Shanghai Collaborative Innovation Program on Regenerative Medicine and Stem Cell Research (2019CXJQ01), Innovative Research Team of High-level Local Universities in Shanghai, Guangci Distinguished Young Scholars Training Program (GCQN-2019-B17), and the Natural Science Foundation of Shanghai, China (19ZR1432400).

Availability of data and materials

All the data obtained and/or analyzed in the current study were available from the corresponding authors on reasonable request. 


\section{Ethics approval and consent to participate}

This study was approved by Ethnics Committee of Sun Yat-sen University Cancer Center and Ruijin Hospital affiliated to Shanghai Jiao Tong University School of Medicine.

\section{Consent for publication}

All authors give consent for the publication of manuscript in Molecular Cancer.

\section{Competing interests}

The authors declare no competing financial interests.

\section{Author details}

${ }^{1}$ Department of Hematologic Oncology, Sun Yat-sen University Cancer Center, Guangzhou 500020, China. ${ }^{2}$ State Key Laboratory of Oncology in South China, Collaborative Innovation Center for Cancer Medicine, 651 Dongfeng East Road, Guangzhou 500020, China. ${ }^{3}$ The University of Texas MD Anderson Cancer Center UTHealth Graduate School of Biomedical Sciences, Houston, TX 77030, USA. ${ }^{4}$ National Research Center for Translational Medicine, Ruijin Hospital affiliated to Shanghai Jiao Tong University School of Medicine, Shanghai 200025, China. ${ }^{5}$ Department of Hematology, Peking University Shenzhen Hospital, Shenzhen 518036, China. ${ }^{6}$ Department of Hematology, Guangzhou Women and Children's Medical Center, Guangzhou Medical University, Guangzhou 510623, China. ${ }^{7}$ State Key Laboratory of Medical Genomics, Shanghai Institute of Hematology, Ruijin Hospital affiliated to Shanghai Jiao Tong University School of Medicine, Shanghai 200025, China. ${ }^{8}$ Department of Hematology, Rui-Jin Hospital North, Shanghai Jiao Tong University School of Medicine, Shanghai 201800, China.

Received: 27 July 2020 Accepted: 21 December 2020

Published online: 05 January 2021

\section{References}

1. Huntington ND, Cursons J, Rautela J. The cancer-natural killer cell immunity cycle. Nat Rev Cancer. 2020;20(8):437-54.

2. Liu E, Marin D, Banerjee P, Macapinlac HA, Thompson P, Basar R, et al. Use of CAR-transduced natural killer cells in CD19-positive lymphoid tumors. N Engl J Med. 2020;382(6):545-53.

3. Djaoud Z, Parham P. HLAs, TCRs, and KIRs, a triumvirate of human cellmediated immunity. Annu Rev Biochem. 2020;89:717-39.

4. Schetelig J, Baldauf H, Heidenreich F, Massalski C, Frank S, Sauter J, et al. External validation of models for KIR2DS1/KIR3DL1-informed selection of hematopoietic cell donors fails. Blood. 2020;135(16):1386-95.

5. Tang L, Wu J, Li CG, Jiang HW, Xu M, Du M, et al. Characterization of immune dysfunction and identification of prognostic immune-related risk factors in acute myeloid leukemia. Clin Cancer Res. 2020;26(7):1763-72.

6. van Galen P, Hovestadt V, Wadsworth Ii MH, Hughes TK, Griffin GK, Battaglia $\mathrm{S}$, et al. Single-cell RNA-Seq reveals AML hierarchies relevant to disease progression and immunity. Cell. 2019;176(6):1265-81 e24.

7. Cooley S, Parham P, Miller JS. Strategies to activate NK cells to prevent relapse and induce remission following hematopoietic stem cell transplantation. Blood. 2018;131(10):1053-62.

8. Dong W, Wu X, Ma S, Wang Y, Nalin AP, Zhu Z, et al. The mechanism of anti-PD-L1 antibody efficacy against PD-L1-negative tumors identifies NK cells expressing PD-L1 as a Cytolytic effector. Cancer Discov. 2019;9(10): 1422-37.

9. Viant C, Guia S, Hennessy RJ, Rautela J, Pham K, Bernat C, et al. Cell cycle progression dictates the requirement for BCL2 in natural killer cell survival. J Exp Med. 2017;214(2):491-510.

10. DiNardo CD, Wei AH. How I treat acute myeloid leukemia in the era of new drugs. Blood. 2020;135(2):85-96.

\section{Publisher's Note}

Springer Nature remains neutral with regard to jurisdictional claims in published maps and institutional affiliations.

\section{Ready to submit your research? Choose BMC and benefit from:}

- fast, convenient online submission

- thorough peer review by experienced researchers in your field

- rapid publication on acceptance

- support for research data, including large and complex data types

- gold Open Access which fosters wider collaboration and increased citations

- maximum visibility for your research: over $100 \mathrm{M}$ website views per year

At BMC, research is always in progress.

Learn more biomedcentral.com/submissions 